\title{
Response of the Inertial Recirculation to Intensified Stratification in a Two-Layer Quasigeostrophic Ocean Circulation Model
}

\author{
SHANTONG SUN AND LIXIN WU \\ Physical Oceanography Laboratory, Ocean University of China, Qingdao, China \\ Bo QIU \\ Department of Oceanography, University of Hawaii at Manoa, Honolulu, Hawaii
}

(Manuscript received 22 June 2012, in final form 18 March 2013)

\begin{abstract}
Previous observation and model studies show that the upper-ocean stratification is enhanced under global warming (Capotondi et al.; Cravatte et al.; Deser et al., etc.). The response of the recirculation, which is associated with the western boundary current (WBC) jet extension and significantly increases its transport, to the intensified stratification, is studied in a two-layer quasigeostrophic ocean circulation model. It is found that the barotropic transport of the circulation first increases with stratification but then decreases as a result of saturation of the surface-layer circulation intensity when the stratification exceeds a threshold. PV budget analysis indicates that the saturation is caused by the increased intergyre transport of relative potential vorticity resulting from the intensified variability of the jet location. Both the barotropic instability and bifurcation mechanisms contribute to the intensified variability of the jet location. Because of barotropic instability, eddies are generated in the confluence region of the WBCs and advected eastward, causing the variability of the jet location. With increased stratification, the surface-layer circulation is strengthened and the barotropic instability is intensified. As a result, the surface flow becomes more variable with excessive eddies and intense variability of the jet. With the increasing stratification, three regimes, each marked by its own variation of the jet location, emerge owing to the successive system bifurcations. In the last two regimes, variability of the jet location is further enhanced by frequent switches among the different dynamic states on multidecadal time scales.
\end{abstract}

\section{Introduction}

The strong recirculation, which flanks the exit region of the western boundary currents (WBCs) and marks the most prominent feature of the wind-driven circulation (Schmitz 1980; Hogg 1983; Pedlosky 1987, 1996), can substantially increases the transport of a WBC (Schmitz 1980) and has been a classical topic since the pioneering work of Fofonoff (1954). The formation mechanisms for the recirculation have been proposed in many previous studies and can be divided into three categories (Waterman and Jayne 2009), that is, the inertial theory in which the recirculation arises from the timemean advection of potential vorticity (PV) by the WBC

Corresponding author address: Shantong Sun, Physical Oceanography Laboratory, Ocean University of China, 238 Songling Road, Qingdao 266100, China.

E-mail: silencesst@ouc.edu.cn
(Marshall and Nurser 1986; Marshall and Marshall 1992; Cessi 1988; Ierley 1987), the eddy-driven theory in which the time-mean recirculation emerges from the eddy-mean flow and eddy-eddy interactions (Cessi 1988; Berloff 2005; Qiu et al. 2008), and the unstable jet theory in which the recirculation is generated from the unstable jet where both eddy effect and inertial effect can play a role (Jayne et al. 1996; Jayne and Hogg 1999; Beliakova 1998).

In addition to the formation mechanisms of the recirculation, the temporal variability and multiple equilibrium solutions of the gyre circulation have also been examined since the mid-1990s. By either increasing the external forcing or decreasing the dissipation in numerical experiments, it was found that the circulation undergoes different regimes, characterized by the emergence of low-frequency temporal variability or multiple solutions, through successive system bifurcations (Jiang et al. 1995; Chang et al. 2001; Cessi and Ierley 1995; 
Qiu and Miao 2000; Pierini et al. 2009; Dijkstra 2000; Dijkstra and Ghil 2005). The interaction between eddies and the mean flow was proposed to account for the low-frequency variability of the gyre circulation, which was usually considered as the result of Hopf bifurcation within the scope of dynamical system theory (Berloff et al. 2007; McCalpin and Haidvogel 1996). For example, McCalpin and Haidvogel (1996) concluded in their study that the low-frequency variability, marked by frequent transitions among multiple states, is due to the interaction between the WBC and eddies formed in the exit region of the eastward jet (EJ) and moving westward into the western boundary area.

Besides the effects of the changing forcing and dissipation, the recirculation can also be affected significantly by the changing stratification. It is relevant to understand the effect of stratification on the WBC jet and recirculation behavior, since previous observation and model studies showed that the upper-ocean stratification has enhanced in the ocean (Capotondi et al. 2012; Cravatte et al. 2009; Deser et al. 2010, etc.). Using a two-layer quasigeostrophic model and weak nonlinear analysis, Liu (1997, hereafter L97) investigated the influence of stratification on the inertial recirculation and found that the barotropic transport is intensified in the presence of a shallower thermocline or a stronger stratification, because of an enhanced PV advection through baroclinic-barotropic interaction. This study extends the work of L97 from the weak nonlinear to the strong nonlinear regime by exploring systematically the variation of the circulation as a result of increasing stratification. The mechanisms involved are diagnosed in terms of PV dynamics.

This paper is organized as follows. Section 2 presents the model configuration and the basic results on the variations of the circulation intensity with stratification. The PV budget analysis of the gyre circulation is provided in section 3 . Section 4 attempts to interpret the recirculation variations in terms of the jet instability and eddy-mean flow interaction. Section 5 is aimed to unravel the low-frequency variability of the system from bifurcation analysis. Summary and additional discussions are given in section 6 .

\section{Model and results}

\section{a. Model configuration and methods}

A two-layer quasigeostrophic ocean circulation model is used in this study. The governing equations for the two dynamical isopycnal layers are as follows (Pedlosky 1987; Berloff et al. 2007):

$$
\begin{aligned}
& \frac{\partial q_{1}}{\partial t}+J\left(\psi_{1}, q_{1}\right)=\frac{f_{0}}{H_{1}} W+\nu \nabla^{4} \psi_{1} \quad \text { and } \\
& \frac{\partial q_{2}}{\partial t}+J\left(\psi_{2}, q_{2}\right)=\nu \nabla^{4} \psi_{2}-r \nabla^{2} \psi_{2} .
\end{aligned}
$$

Here, subscript 1 and 2 indicate the surface and bottom layer, respectively, $q_{i}$ represents the potential vorticity, $\psi_{i}$ represents the streamfunction, and $H_{i}$ represents the mean layer thickness of layer $i(i=1,2)$. Lateral friction with a constant lateral dissipation coefficient $\nu$ and bottom friction in Rayleigh form with a constant coefficient $r$ are both included as the dissipation mechanisms. The potential vorticity for the surface and bottom layer are defined as follows:

$$
\begin{aligned}
& q_{1}=\nabla^{2} \psi_{1}+F_{1}\left(\psi_{2}-\psi_{1}\right)+\beta y, \\
& q_{2}=\nabla^{2} \psi_{2}+F_{2}\left(\psi_{1}-\psi_{2}\right)+\beta y, \quad \text { and } \\
& F_{1}=\frac{f_{0}^{2}}{g^{\prime} H_{1}}, \quad F_{2}=\frac{f_{0}^{2}}{g^{\prime} H_{2}},
\end{aligned}
$$

where $g^{\prime}=g\left(\rho_{2}-\rho_{1}\right) / \rho_{0}$ represents the reduced gravity parameter, $\rho_{1}, \rho_{2}$, and $\rho_{0}$ indicate the density for the surface layer, the bottom layer, and the reference state, respectively. Note that only the thickness terms in Eq. (2), that is, $F_{i}\left(\psi_{3-i}-\psi_{i}\right)$, are explicitly connected to stratification. Accordingly, we divide the PV into three parts: the relative potential vorticity (RPV) $\nabla^{2} \psi_{1}$, the thickness potential vorticity (TPV) $F_{i}\left(\psi_{3-i}-\psi_{i}\right)$, and the planetary potential vorticity $\beta y$ (Pedlosky 1987). The wind forcing $W$ is prescribed in an antisymmetric form with respect to the midbasin axis:

$$
W=-w_{0} \sin \left(2 \pi y / L_{y}\right),
$$

where $w_{0}$ indicates the amplitude of the Ekman pumping velocity.

The equations are integrated using a leapfrog timestepping algorithm, and the elliptical equation is solved to derive streamfunction using the fast Poisson Solver. The boundary conditions are chosen as a partial-slip lateral boundary condition, that is,

$$
\alpha \frac{\partial^{2} \psi_{i}}{\partial n^{2}}-\frac{\partial \psi_{i}}{\partial n}=0,
$$

and no normal flow condition, that is,

$$
\psi_{i}=\psi_{i}(t)
$$

Here, $\alpha=16 \mathrm{~km}$ lies between the no-slip lateral boundary condition $(\alpha=0 \mathrm{~km})$ and the free-slip boundary 
condition $(\alpha \rightarrow \infty)$. The partial derivatives in Eq. (4a) are taken normal to the lateral boundary. In general, the boundary condition with a smaller $\alpha$ is more efficient in dissipating the potential vorticity input by the external forcing, and will result in a weaker circulation. A detailed discussion on the sensitivity of circulation to the choice of $\alpha$ is provided by Haidvogel et al. (1992) and Berloff et al. (2007). Finally, the mass conservation equation is also incorporated as follows:

$$
\frac{\partial}{\partial t} \int\left(\psi_{1}-\psi_{2}\right) d s=0
$$

where the integration is performed over the entire model domain.

The standard parameters used in the model are listed in Table 1. The spatial resolution is $8 \mathrm{~km}$, which allows resolving mesoscale eddies. After a spinup for 40 years from a state of rest, each case experiment is integrated for another 200 years. We explore in this study by systematically varying the reduced-gravity value over a wide range $\left(0.005-0.11 \mathrm{~m} \mathrm{~s}^{-2}\right.$; stratification of the Atlantic Ocean is around $0.02-0.03 \mathrm{~m} \mathrm{~s}^{-2}$ ), which is artificially large for the real ocean stratification to reach but is meaningful in an idealized theoretical study.

The barotropic streamfunction is defined by $\psi_{B}=$ $\left(H_{1} \psi_{1}+H_{2} \psi_{2}\right) / H$, where $H=H_{1}+H_{2}$ is the total thickness of the two layers. In this study, we focus on the variation of the maximum transport of the double gyre, which reflects the strength of the inertial recirculation and is defined by

$$
T_{\mathrm{dg}, i}=\left(\psi_{\mathrm{st}, i}-\psi_{\mathrm{sp}, i}\right) \times H_{i},
$$

where $\psi_{\text {st }}$ indicates the maximum positive streamfunction of the subtropical gyre, and $\psi_{\mathrm{sp}}$ represents the minimum negative streamfunction of the subpolar gyre. If the surface-layer (bottom/barotropic) circulation is of concern, the surface-layer (bottom/barotropic) streamfunction is used with $H_{i}$ given by $H_{1}\left(H_{2} / H\right)$. The inertial recirculation intensity is of great importance here since it reflects the strength of the WBC systems, which plays a vital role in the balance of momentum and energy of the ocean circulation (Waterman and Jayne 2009; Pedlosky 1987, 1996).

Without being specified otherwise, the time averaging in this study is taken over the total 200 model years. It is worth noting that the 200-yr average is long enough if we are studying the variation of the circulation intensity on decadal time scales and shorter, but not if we are focusing on the time-mean circulation pattern for which the reason will be illustrated in the following sections.
TABLE 1 . The model parameters used in the standard case. The reduced gravity $g^{\prime}$ will be varied from 0.005 to $0.11 \mathrm{~m} \mathrm{~s}^{-2}$ and the value given here is the referenced value that resembles the observation.

\begin{tabular}{llll}
\hline \hline$f_{0}$ & $1.0 \times 10^{-4} \mathrm{~s}^{-1}$ & $\beta$ & $1.3 \times 10^{-11}(\mathrm{~m} \mathrm{~s})^{-1}$ \\
$\rho_{0}$ & $1.0 \times 10^{3} \mathrm{~kg} \mathrm{~m}^{-3}$ & $g^{\prime}$ & $0.03 \mathrm{~m} \mathrm{~s}^{-2}$ \\
$H_{1}$ & $1000 \mathrm{~m}$ & $H_{2}$ & $3000 \mathrm{~m}$ \\
$\nu$ & $140 \mathrm{~m}^{2} \mathrm{~s}^{-1}$ & $r$ & $1.0 \times 10^{-7} \mathrm{~s}^{-1}$ \\
$L_{\mathrm{x}}$ & $2400 \mathrm{~km}^{-6} \mathrm{~m} \mathrm{~s}^{-1}$ & $L_{y}$ & $2000 \mathrm{~km}$ \\
$w_{0}$ & $2.0 \times 10^{-0} \mathrm{~m}$ & & \\
\hline
\end{tabular}

In addition, we define the location of the EJ where the surface-layer zonal velocity, averaged zonally over $0<x<400 \mathrm{~km}$, reaches maximum.

\section{b. Results}

With an antisymmetric forcing, the 200-yr-mean barotropic circulation for $g^{\prime}=0.03 \mathrm{~m} \mathrm{~s}^{-2}$, which is around the real ocean stratification, displays an antisymmetric pattern with counterrotating gyres of the same strength as is illustrated in Fig. 1. For comparison, the barotropic circulation patterns for $g^{\prime}=0.01,0.05,0.07,0.09$, and $0.11 \mathrm{~m} \mathrm{~s}^{-2}$ are also presented in Fig. 1 .

Variations of the maximum transport with stratification are depicted in Fig. 2. It is found that the maximum barotropic transport increases with stratification for $g^{\prime}$ less than $0.05 \mathrm{~m} \mathrm{~s}^{-2}$, but decreases when $g^{\prime}$ increases further. In the surface layer, the transport increases with stratification when it is less than $0.05 \mathrm{~m} \mathrm{~s}^{-2}$, but then saturates as $g^{\prime}$ exceeds $0.05 \mathrm{~m} \mathrm{~s}^{-2}$. In the bottom layer, the transport decreases systematically with increasing $g^{\prime}$. However, this decrease in the bottomlayer transport is relatively weak when $g^{\prime}$ is less than $0.05 \mathrm{~m} \mathrm{~s}^{-2}$. Another point to note is that the variation of the zonal penetration scale (Marshall and Marshall 1992) of the zonal jet shows a similar pattern with that of the surface-layer intensity; specifically, it increases first and then reaches saturation in the stronger stratification cases (Fig. 1).

Accordingly, variations of the recirculation with stratification can be divided into two regimes. ${ }^{1}$ Within the weak stratification regime $\left(g^{\prime} \leq 0.05 \mathrm{~m} \mathrm{~s}^{-2}\right)$, variation of the circulation intensity is consistent with the result in L97. However, within the strong stratification regime $\left(g^{\prime}>0.05 \mathrm{~m} \mathrm{~s}^{-2}\right)$, the barotropic circulation

\footnotetext{
${ }^{1} \mathrm{We}$ assume the real-world ocean is located in the weak stratification regime considering that the observed stratification is around $0.02 \mathrm{~m} \mathrm{~s}^{-2}$. However, this statement requires further studies to make it certain, given that the regime partition is strongly dependent on the choice of model parameters.
} 

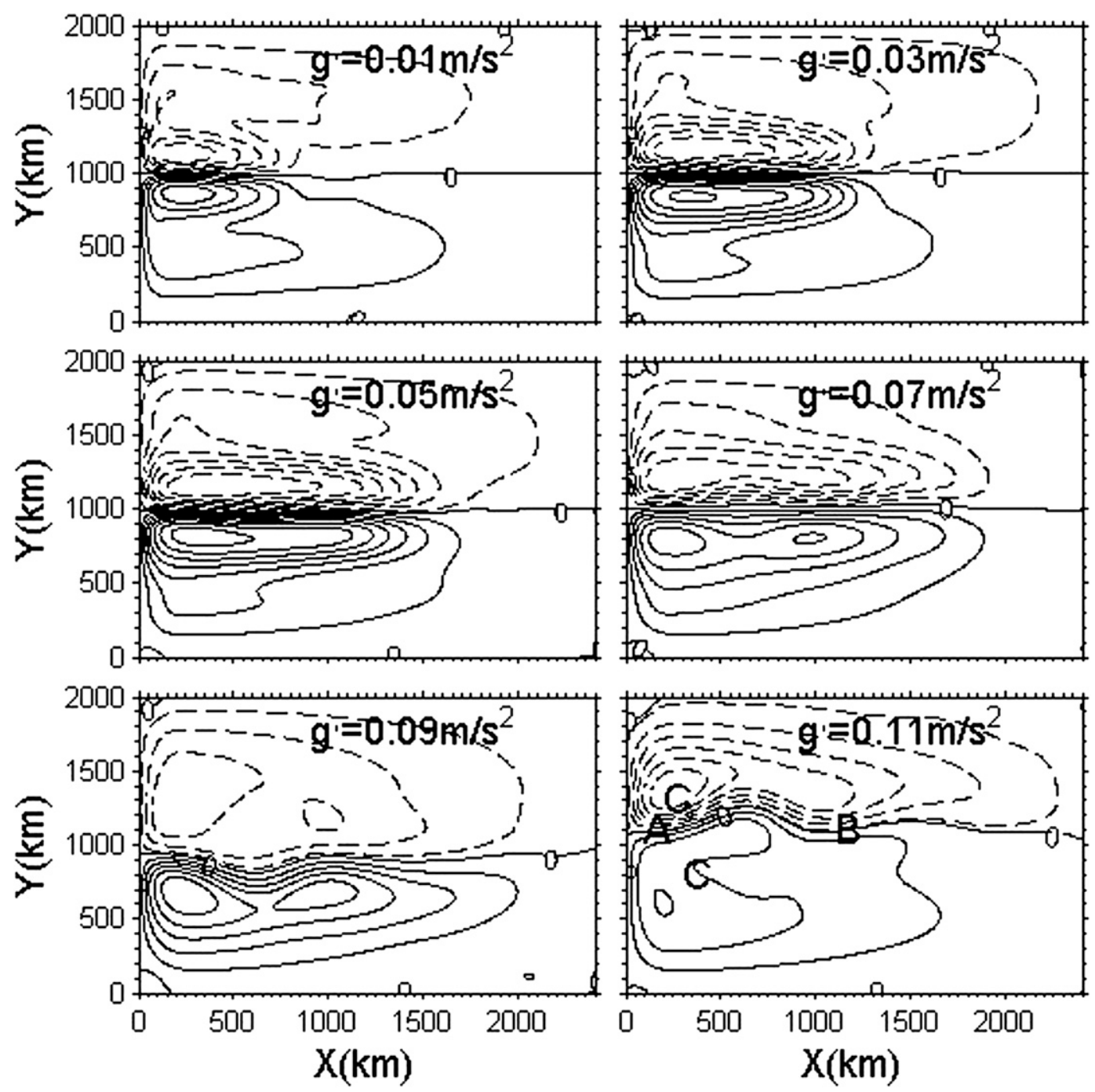

FIG. 1. The 200-yr-mean barotropic streamfunction for $g^{\prime}$ equal to (top left) 0.01 , (top right) 0.03 , (middle left) 0.05, (middle right) 0.07, (bottom left) 0.09 , and (bottom right) $0.11 \mathrm{~m} \mathrm{~s}^{-2}$. The solid (dashed) lines indicate positive (negative) values and subtropical (subpolar) gyre. The contour interval is 3000 . (bottom right) Capital A defines the confluence region of the WBCs and the upstream region of the jet $(x: 0 \sim 100 \mathrm{~km})$; capital B defines the exit region of the recirculation and the downstream region of the jet; capital $\mathrm{C}$ defines the recirculation region.

intensity is reduced at an enhanced stratification. It is verified through sensitivity experiments (not shown) by varying the basin's zonal scale $L_{x}$ that the existence of the two-regime pattern is independent of $L_{x}$, but the switch point, that is, the stratification where the second regime begins, is shifted to a lower (higher) $g^{\prime}$ value when the basin's zonal scale is decreased (increased). Finally, we note that the sensitivity experiments using the no-slip boundary condition (not shown) also capture the two-regime pattern in the similar way, with the switch point shifted to a higher $g^{\prime}$ value. It requires attention that this dependence of the circulation on $L_{x}$ and boundary condition is not fully understood yet and left for future study.

\section{PV dynamics of the recirculation}

In this section, we first attempt to interpret the response of the circulation to the varying stratification by exploring the PV dynamics of the recirculation. By decomposing the total streamfunction into the mean and anomaly state as $\psi_{i}=\Psi_{i}+\varphi_{i}$ and substituting it into Eq. (1), then taking time averaging over Eq. (1) and neglecting the timedependent terms, we obtain the following equations: 

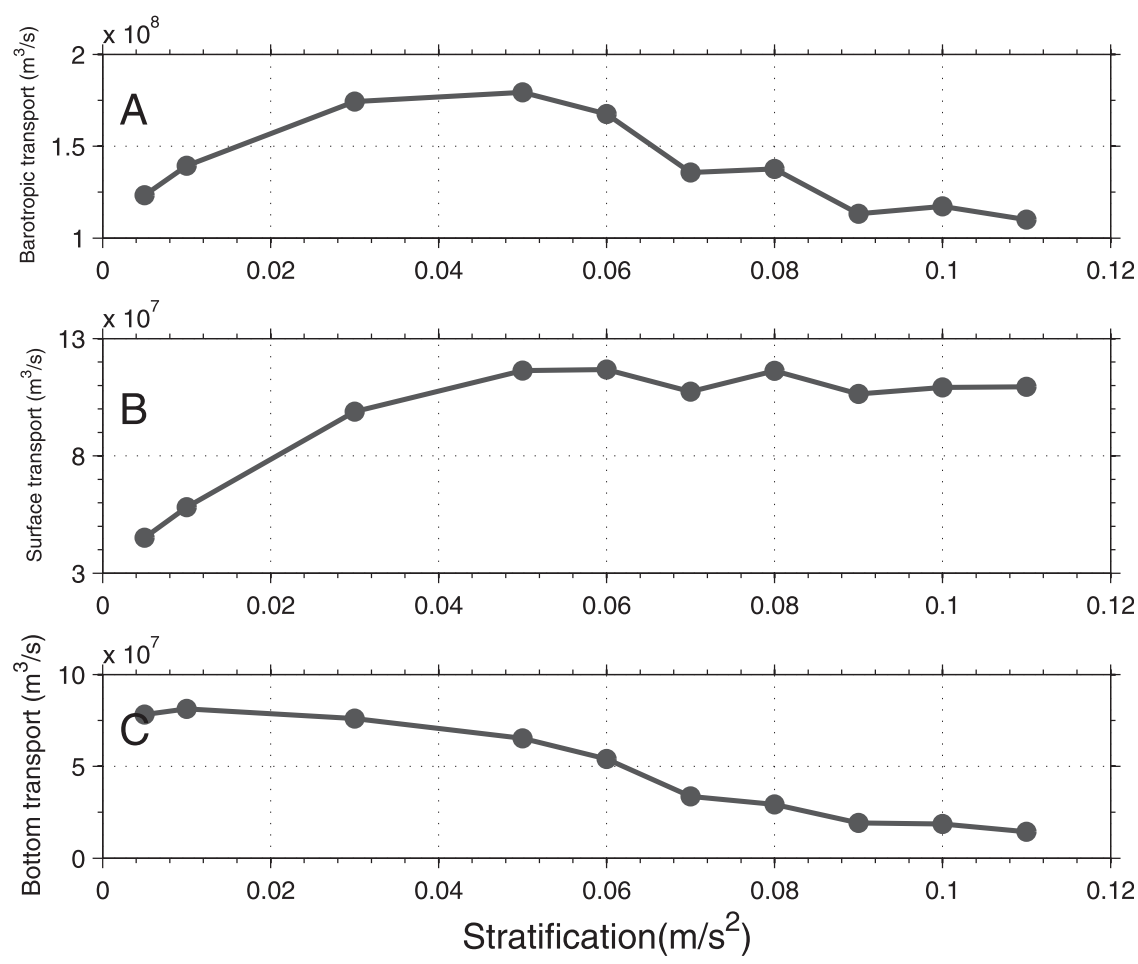

FIG. 2. Variations of the (a) maximum barotropic, (b) surface-layer, and (c) bottom-layer transport for the 200-yr-mean circulation. The abscissa indicates the stratification $\left(\mathrm{m} \mathrm{s}^{-2}\right)$ and the ordinate denotes the maximum transport $\left(\mathrm{m}^{3} \mathrm{~s}^{-1}\right)$.

$$
\left\{\begin{array}{c}
J\left(\Psi_{1}, \nabla^{2} \Psi_{1}\right)+\overline{J\left(\varphi_{1}, \nabla^{2} \varphi_{1}\right)}+\frac{f_{0}^{2}}{g^{\prime} h_{1}} J\left(\Psi_{1}, \Psi_{2}\right)+\frac{f_{0}^{2}}{g^{\prime} h_{1}} \overline{J\left(\varphi_{1}, \varphi_{2}\right)}+\beta \frac{\partial \Psi_{1}}{\partial x}=f \frac{W}{H}+A_{H} \nabla^{4} \Psi_{1} \\
J\left(\Psi_{2}, \nabla^{2} \Psi_{2}\right)+\overline{J\left(\varphi_{2}, \nabla^{2} \varphi_{2}\right)}+\frac{f_{0}^{2}}{g^{\prime} h_{2}} J\left(\Psi_{2}, \Psi_{1}\right)+\frac{f_{0}^{2}}{g^{\prime} h_{2}} \overline{J\left(\varphi_{2}, \varphi_{1}\right)}+\beta \frac{\partial \Psi_{2}}{\partial x}=A_{H} \nabla^{4} \Psi_{2}-r \nabla^{2} \Psi_{2}
\end{array} .\right.
$$

The overline in Eq. (7) indicates time average. Note that $J\left(\psi_{i}, \eta\right)=\boldsymbol{\nabla} \cdot\left(\eta \mathbf{V}_{i}\right)$, the Jacobian terms in Eq. (7) are, therefore, in forms of divergence. In the surface layer, Eq. (7a) indicates that the local PV balance is maintained by the divergence of RPV by the mean flow and eddies, the divergence of TPV by the mean flow and eddies, the planetary vorticity advection, the wind forcing, and the lateral dissipation. Here, we concentrate on the roles of the divergence terms in driving the recirculation because of its known inertial nature (It is confirmed by diagnosis that the lateral dissipation and wind forcing in the recirculation area are smaller than these nonlinear terms by at least an order of magnitude; not shown).

The spatial distributions of the divergence of TPV and RPV for the surface layer are illustrated in Fig. 3. It is shown that, overall, the divergence term of TPV by the mean flow and eddies has signs opposite to the divergence term of RPV by the mean flow and eddies in the
WBC and recirculation regions. The exception is in the upstream region of the EJ where the divergence of RPV by eddies resembles the divergence of TPV by eddies. It is worth noting that the divergence of RPV is almost an order of magnitude larger than TPV in the recirculation region. This result points to the importance of relative vorticity in the dynamics of the recirculation and is consistent with the previous findings that the nonlinear terms work to force the recirculation (Waterman and Jayne 2009; Jayne and Hogg 1999).

To clarify how each component affects the inertial recirculation, the linear flow driven by the divergence terms are calculated using Eq. (8):

$$
D+\beta \frac{\partial \psi}{\partial x}=0
$$

where $D$ denotes the divergence of TPV or RPV by the mean flow or eddies. The streamfunction $\psi$ obtained by 

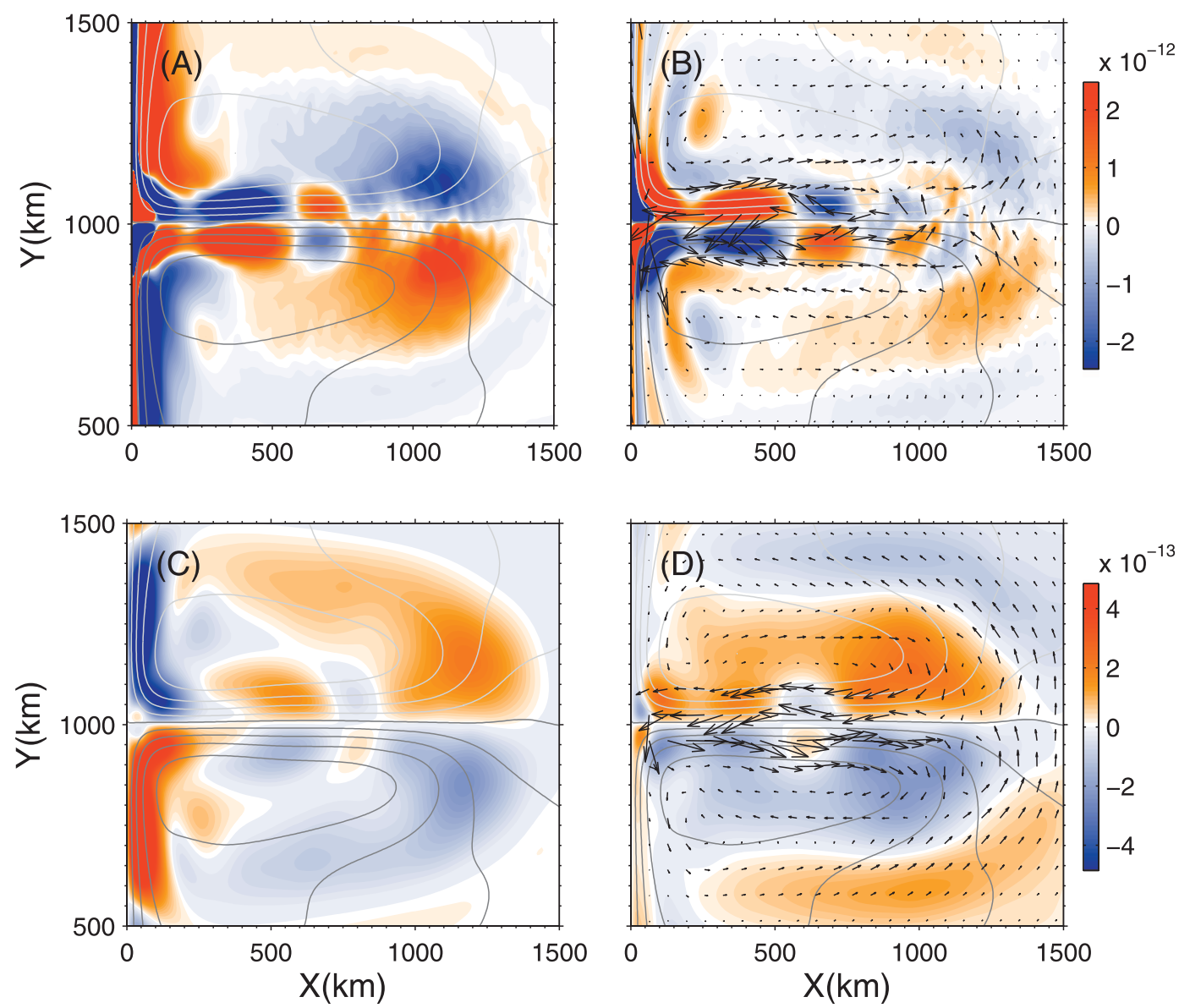

FIG. 3. The spatial distribution of the divergence of RPV by (a) mean flow and (b) eddies, the divergence of TPV by (c) mean flow and (d) eddies in the surface-layer inertial recirculation region $\left(g^{\prime}=0.03 \mathrm{~m} \mathrm{~s}^{-2}\right)$. Note that the positive (negative) value of the divergence terms tends to strengthen (weaken) anticyclone flow or weaken (strengthen) cyclone flow. The contours indicate the time-mean streamfunction of surface layer. Arrows in (b) and (d) indicate the time-mean eddy flux of RPV and TPV, respectively.

integrating Eq. (8) is provided in Fig. 4. It shows that the sum of these four terms in Fig. 4 agrees well with the modeled recirculation (not shown). Accordingly, within the recirculation area, the streamfunction associated with the RPV divergence has the same sign as the timemean recirculation (Figs. 4a,b), while the streamfunction associated with the TPV divergence has the opposite sign to the time-mean recirculation (Figs. 4c,d). These results imply that the surface-layer recirculation is largely driven by the divergence of RPV, with the divergence of TPV playing a damping role. In the upstream region of the EJ, the streamfunction shown in Fig. 4b displays patterns that are opposite to the recirculation region, suggesting that the divergence of RPV associated with eddies tends to slacken the midlatitude jet, which is expected for barotropic instability.

It can be inferred that, with the intensification of stratification, the efficiency of the divergence of TPV in weakening the surface-layer recirculation will be reduced [ $F_{i}$ in Eq. (2) is reduced], that is, the momentum transfer from the surface layer to the bottom layer will be restrained. In fact, this statement is confirmed by the reduction of the transport of the circulation driven by the divergence of TPV, with the increase of $g^{\prime}$ (not shown). Therefore, the surface-layer recirculation, in this case, will develop both in size and in intensity while the bottom-layer recirculation will be weakened. This is consistent with the recirculation changes within the weak nonlinear regime shown in Fig. 2.

As is illustrated in Eq. (7b), only the TPV terms serve as external forcing for the bottom layer. The efficiency of this forcing is sensitive to both the circulation intensity and the stratification. An increased stratification tends to weaken the forcing for the bottom layer while the intensification of the surface-layer flow, to some extent, counteracts this effect. As a consequence, the 

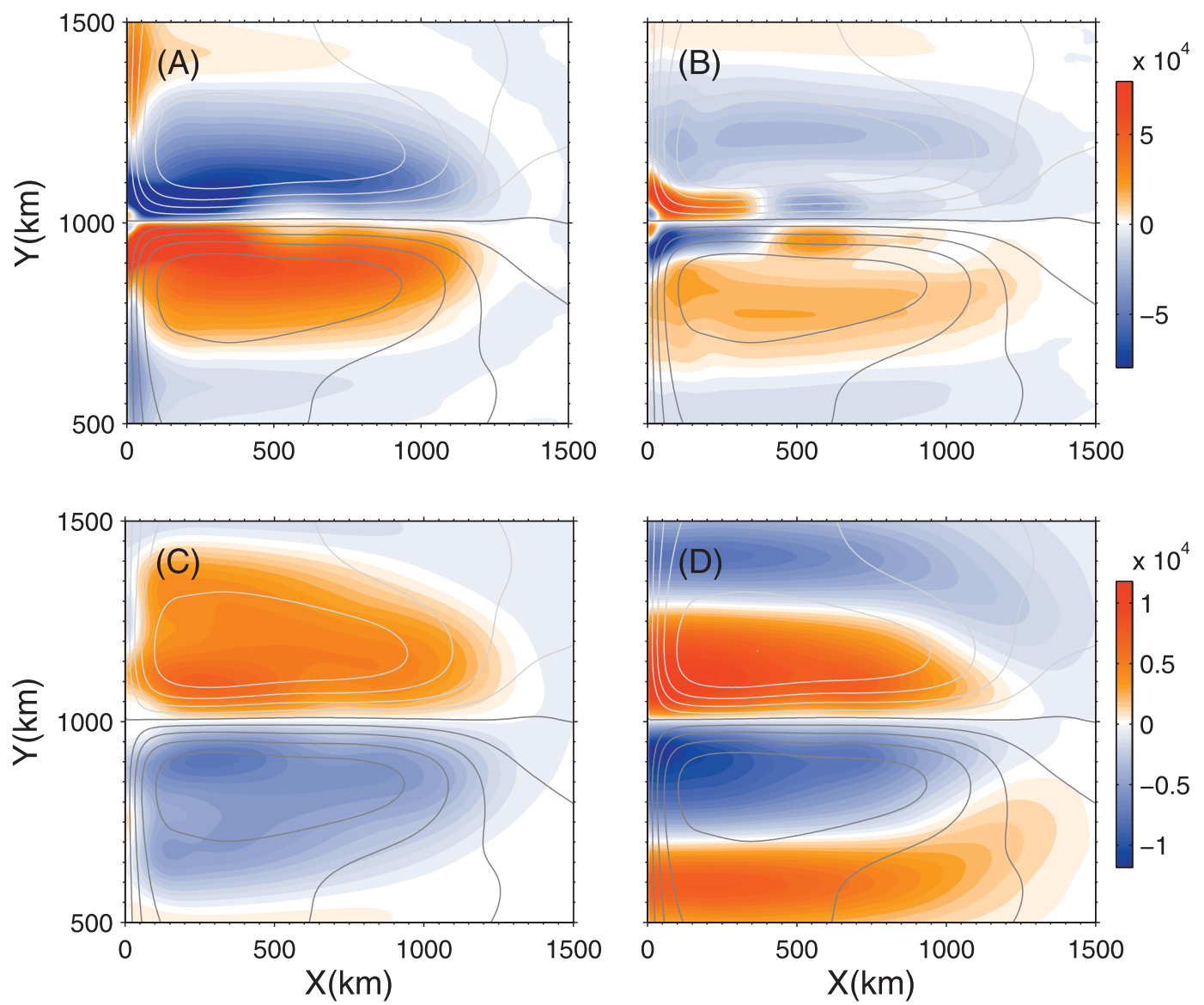

FIG. 4. The flow driven by the divergence terms corresponding to Fig. 3 (positive/negative value denotes anticyclone/ cyclone flow; the contours indicate the time-mean streamfunction for the surface-layer recirculation; $g^{\prime}=0.03 \mathrm{~m} \mathrm{~s}^{-2}$ ).

bottom circulation transport shows a weak decrease in the weak stratification regime where the surface circulation strengthens substantially, but a sharp decrease in the strong stratification regime where the surface-layer circulation saturates.

In the next, we attempt to explore the mechanisms responsible for the saturation of the surface-layer circulation as the stratification becomes stronger. Several previous studies have cast doubt on the simplistic judgment that the circulation will strengthen systematically at an intensified external forcing or a weakened dissipation. Using a 1.5-layer QG model with a no-slip boundary condition, McCalpin and Haidvogel (1996) found that, within some specific parameter range, the total energy of the gyre circulation decreases when the wind forcing becomes stronger because of the intensified interaction between the eddies and the eastward jet. Primeau (1998) also found that the circulation becomes weakened with decreasing dissipation when the lateral friction coefficient becomes lower than a threshold value, although the zonal penetration of the jet extends.
Primeau attributed this phenomenon to the increased intergyre transport of PV. Sheremet et al. (1997) showed that the circulation intensity can reach saturation owing to the emergence of "inertial runaway," which marks the abrupt increase of transport and disappearance of east-west asymmetry of the ocean circulation, when the Reynolds number exceeds a critical value (Pedlosky 1987, 1996).

Since the time-mean recirculation fails to reach the eastern boundary (Fig. 1), we can rule out the relevance of the inertial runaway processes in our model runs. In the following, we will examine the possibility to which the intergyre transport of PV is responsible for the saturation of the surface-layer circulation within the strong stratification regime. And it will be shown in the next section that the intensified eddy-jet interaction is one important factor that account for the increased intergyre transport of PV.

By integrating Eq. (7a) over the subtropical and subpolar gyres bounded by some time-mean streamlines, the terms except for the lateral dissipation related to the 

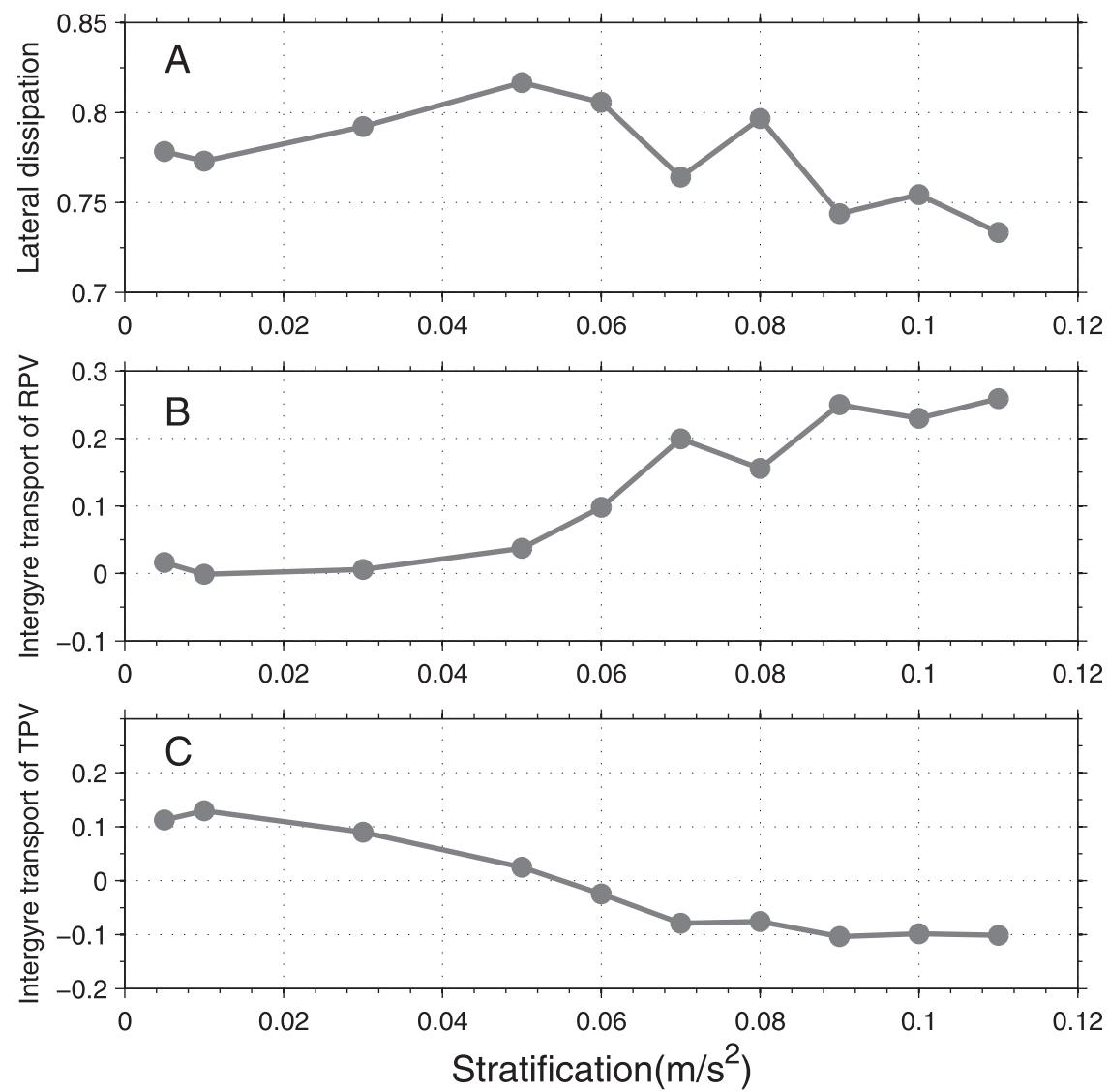

FIG. 5. Variation of (a) the lateral dissipation, (b) the intergyre transport of RPV, and (c) TPV defined in Eq. (9) normalized by the PV input by the wind (unit: 100\%).

time-mean flows vanish. Subtracting the integration of Eq. (7a) over the subpolar gyre (SP) from that over the subtropical gyre (ST), we obtain the following PV budget equation of the double-gyre:

$$
\begin{aligned}
& {\left[\int_{\mathrm{ST}} \overline{J\left(\varphi_{1}, \nabla^{2} \varphi_{1}\right)} d s-\int_{\mathrm{SP}} \overline{J\left(\varphi_{1}, \nabla^{2} \varphi_{1}\right)} d s\right]+\frac{f_{0}^{2}}{g^{\prime} h_{1}}\left[\int_{\mathrm{ST}} \overline{J\left(\varphi_{1}, \varphi_{2}\right)} d s-\int_{\mathrm{SP}} \overline{J\left(\varphi_{1}, \varphi_{2}\right)} d s\right]} \\
& -A_{H}\left(\int_{\mathrm{ST}} \nabla^{4} \Psi_{1} d s-\int_{\mathrm{SP}} \nabla^{4} \Psi_{1} d s\right)=\frac{f}{H}\left(\int_{\mathrm{ST}} W d s-\int_{\mathrm{SP}} W d s\right) .
\end{aligned}
$$

Physically, Eq. (9) states that the PV input by the wind is balanced by the lateral dissipation, the intergyre transport of TPV (T-TPV) and the intergyre transport of RPV (T-RPV). Figure 5 shows the lateral dissipation, T-TPV and T-RPV values as a function of $g^{\prime}$. For simplicity, all values have been normalized by the wind input terms on the rhs of Eq. (9). Overall, in terms of 200-yr average, the PV input by the wind is primarily removed by the lateral dissipation, which accounts for $70 \%-85 \%$ (Fig. 5a). In the weak stratification regime $\left(g^{\prime}<0.05 \mathrm{~m} \mathrm{~s}^{-2}\right)$, both the lateral dissipation and T-RPV increase slightly with stratification, while T-TPV decreases relatively fast. In the strong stratification regime $\left(g^{\prime}>\right.$ $0.05 \mathrm{~m} \mathrm{~s}^{-2}$ ), however, the lateral dissipation decreases from the maximum $83 \%$ at $g^{\prime}>0.05 \mathrm{~m} \mathrm{~s}^{-2}$ to $72 \%$ at $g^{\prime}=$ $0.11 \mathrm{~m} \mathrm{~s}^{-2}$, T-RPV increases dramatically from $5 \%$ to $28 \%$, and T-TPV decreases from $0 \%$ to $-10 \%$. This means that the intergyre transport of TPV contributes to increase the PV different between the gyres, and we assume that this is associated with the meandering of EJ. Note that the sum of these three terms on the lhs of Eq. (9) can only balance $\sim 90 \%$ of the wind input PV. We attribute this to the neglect of the temporal variation in Eq. (9) and errors of numerics. 

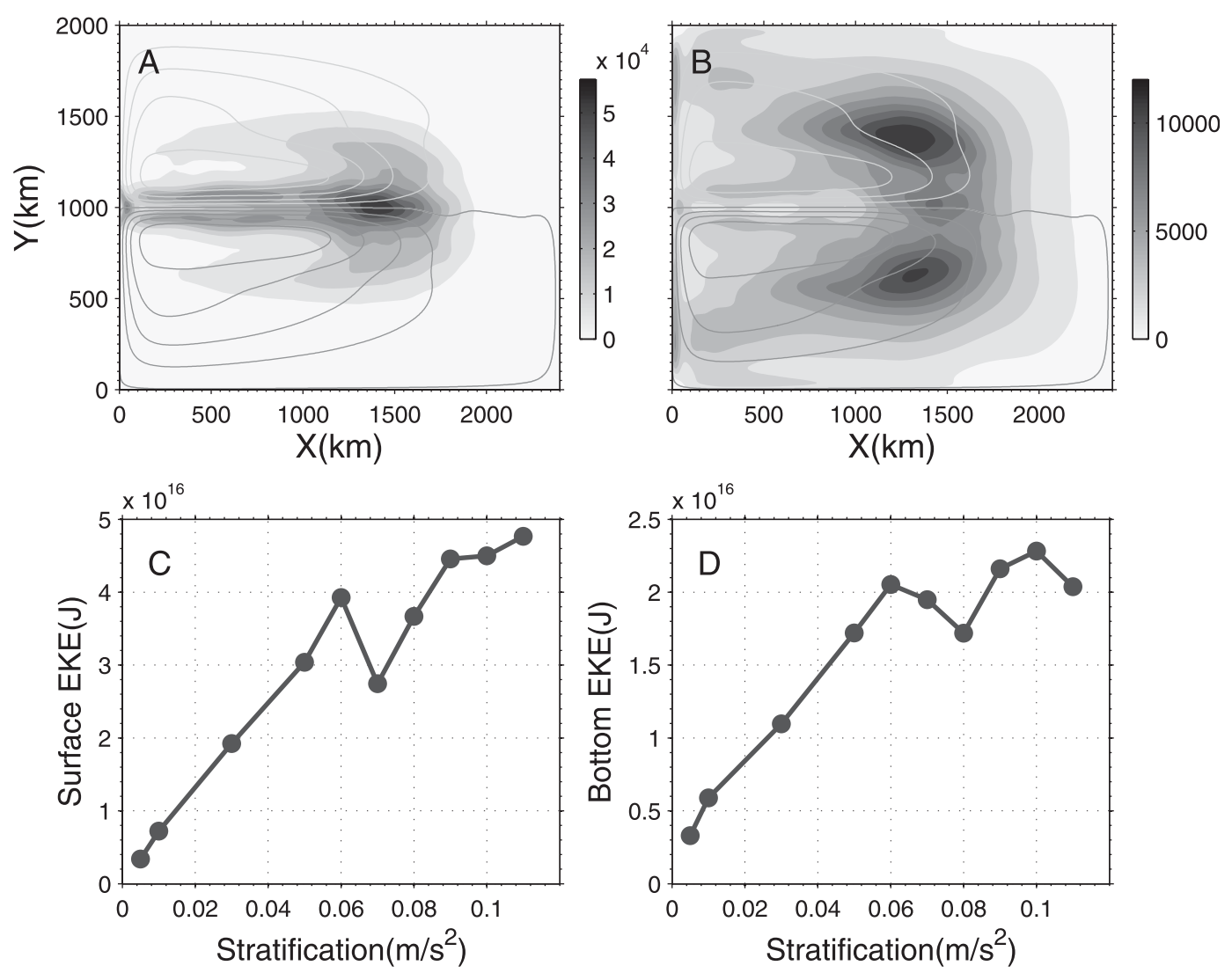

FIG. 6. Spatial distribution of the time-mean eddy kinetic energy for (a) the surface layer and (b) the bottom layer (contours indicate the time-mean surface flow streamfunction); variation of the basin-integrated EKE for (c) the surface layer and (d) the bottom layer with stratification.

We conclude from the PV budget analysis that the saturation of the surface-layer circulation intensity in the large $g^{\prime}$ cases is due to the increased intergyre transport of RPV. Accordingly, a northward (southward) shifted jet is associated with an overshooting subtropical (subpolar) gyre WBC, which transports anomaly negative (positive) RPV into the subpolar (subtropical) gyre, as illustrated in Fig. 3b. The increased intergyre transport of RPV is, therefore, plausible linked to the intensified variability of the jet location.

It should, however, be noted that the diagnosis of the PV budget presents no information about the mechanisms responsible for the intensified variability of the jet location. To clarify the dynamics behind this, we will conduct the instability and bifurcation analyses in the next two sections.

\section{Instability analysis}

By dividing the flow into the mean state and perturbation as in section 3, we can calculate the eddy kinetic energy (EKE):

$$
\mathrm{EKE}_{i}=\frac{1}{2} \rho H_{i}\left|\nabla \phi_{i}\right|^{2}, \quad i=1,2
$$

to illustrate the distribution of eddies and the variability of the current. Considering the time scales for the mesoscale eddies, the time-mean quantities in this section are calculated over the last 20 model years of the first century during the numerical integration. It is worth noting that, by definition, the eddy here is the perturbations from the time-mean state, and must include the jet meanders.

Figures $6 \mathrm{a}$ and $6 \mathrm{~b}$ display the spatial distribution of the surface-layer and bottom-layer time-mean EKE for $g^{\prime}=0.05 \mathrm{~m} \mathrm{~s}^{-2}$. The main feature in the surface layer is that the EKE peaks at the EJ region, especially at the downstream region of the EJ (around $x$ : 1000-1500 km; $y: 800-1200 \mathrm{~km}$ ), which is indicative of the maximum meandering of the jet. In the bottom layer, the EKE reaches its maximum at the eastern edges of the recirculation that flanks the jet. It is noted that the EKE peak in the bottom layer is collocated with that of the surface layer at the downstream region of EJ and is mainly attributed to the existence of eddies that are 

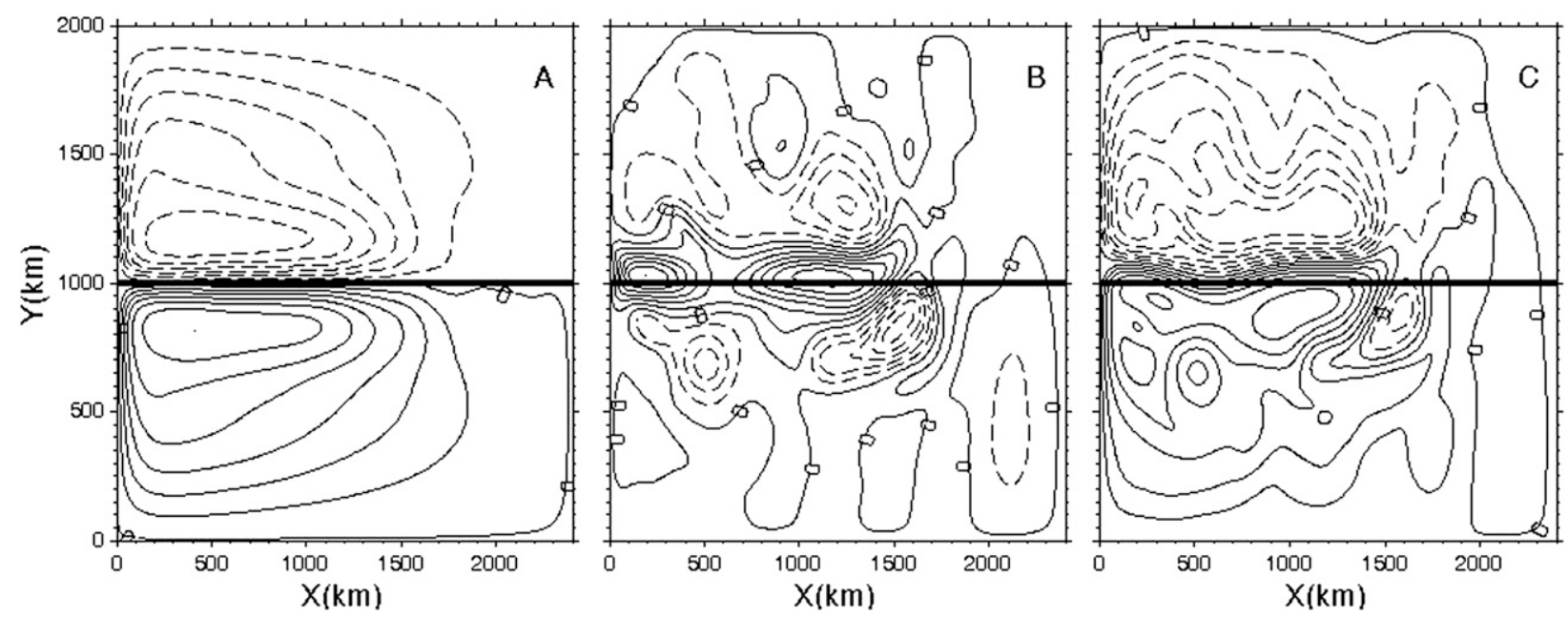

FIG. 7. Contours of (a) the 20-yr-mean surface flow streamfunction, (b) the deviation from the time-mean flow, and (c) the instantaneous streamfunction. Solid (dashed) lines indicate positive (negative) value $\left(g^{\prime}=0.05 \mathrm{~m} \mathrm{~s}^{-2}\right)$. It is shown that, with the emergence of anticyclone eddies along the EJ, the jet is shifted northward.

distinct from jet meanders. The collocation between eddies and the variability of jet location for the surface layer in Fig. 7 suggests that the latter can result from the development of eddies.

By integrating Eq. (10) over the basin, we obtain the basin-integrated EKE for the surface layer and the bottom layer:

$$
\varepsilon_{i}=\int \frac{1}{2} \rho H_{i}\left|\nabla \phi_{i}\right|^{2} d s_{i}, \quad i=1,2
$$

Figures $6 \mathrm{c}$ and $6 \mathrm{~d}$ show the variation of the basinintegrated EKE with stratification and it is seen that both the surface-layer EKE and bottom-layer EKE show almost systematic increase with stratification, indicating that the circulation becomes more variable with excessive eddies and intense variability of the jet when the stratification is increased.

Following Berloff and Meacham (1998), the energy exchange between the basic flow and perturbation due to the work done by the Reynolds stresses $P$ and due to the divergent thickness flux $R$ is as follows:

$$
P_{i}=-H_{i} \overline{\left[\phi_{i x} J\left(\phi_{i}, \Psi_{i x}\right)+\phi_{i y} J\left(\phi_{i}, \Psi_{i y}\right)\right]}, \quad i=1,2 \text { and }
$$

$$
R=-\frac{f_{0}^{2}}{2 g^{\prime}} \overline{\left.\left(\phi_{1}-\phi_{2}\right) J\left[\frac{1}{2}\left(\phi_{1}+\phi_{2}\right), \Psi_{1}-\Psi_{2}\right]\right\}}
$$

respectively. Given the importance of relative potential vorticity in the dynamics of the recirculation as illustrated in section 3 , only the spatial distribution of $P_{1}$ is shown in Fig. 8 for the $\mathrm{g}^{\prime}=0.03,0.05,0.07$, and $0.09 \mathrm{~m} \mathrm{~s}^{-2}$ cases $\left(P_{1}\right.$ is of the same scale with $R$ in the weak stratification regime, but larger than $R$ by an order of magnitude in the strong stratification regime; not shown). Note that only in the recirculation and jet regions does the energy exchange between the mean flow and the perturbation appear prominent, so we merely concentrate on this area. It is found that almost all over the basin $P_{1}$ is positive, and the largest value of $P_{1}$ is concentrated in the vicinity of the jet (Fig. 8). Accordingly, eddies are generated at the upstream region of the jet and advected eastward along the EJ (Figs. 9a,b). Meanwhile, they continuously extract kinetic energy from the shear of mean EJ and grow to maximum at its exit region (Figs. 9c,d; Fig. 6a). Accompanied by the eastward advection of these eddies, the jet will be shifted either south or north from the midbasin axis and contribute to the intergyre transport of PV.

Here, we define the index for barotropic instability and baroclinic instability as the integration of Eq. (12) over the basin:

$$
\begin{aligned}
& I_{P}=-\int H_{1} \overline{\left[\phi_{1 x} J\left(\phi_{1}, \Psi_{1 x}\right)+\phi_{1 y} J\left(\phi_{1}, \Psi_{1 y}\right)\right]} d s \\
& I_{R}=-\int \frac{f_{0}^{2}}{2 g^{\prime}} \overline{\left\{\left(\phi_{1}-\phi_{2}\right) J\left[\frac{1}{2}\left(\phi_{1}+\phi_{2}\right), \Psi_{1}-\Psi_{2}\right]\right\}} d s .
\end{aligned}
$$

Here, $I_{P}$ and $I_{R}$ represent the energy the perturbations extract from the mean-flow owing to barotropic instability and baroclinic instability, respectively. The circulation is subject more likely to baroclinic instability in the weak stratification regime and to barotropic instability in the 

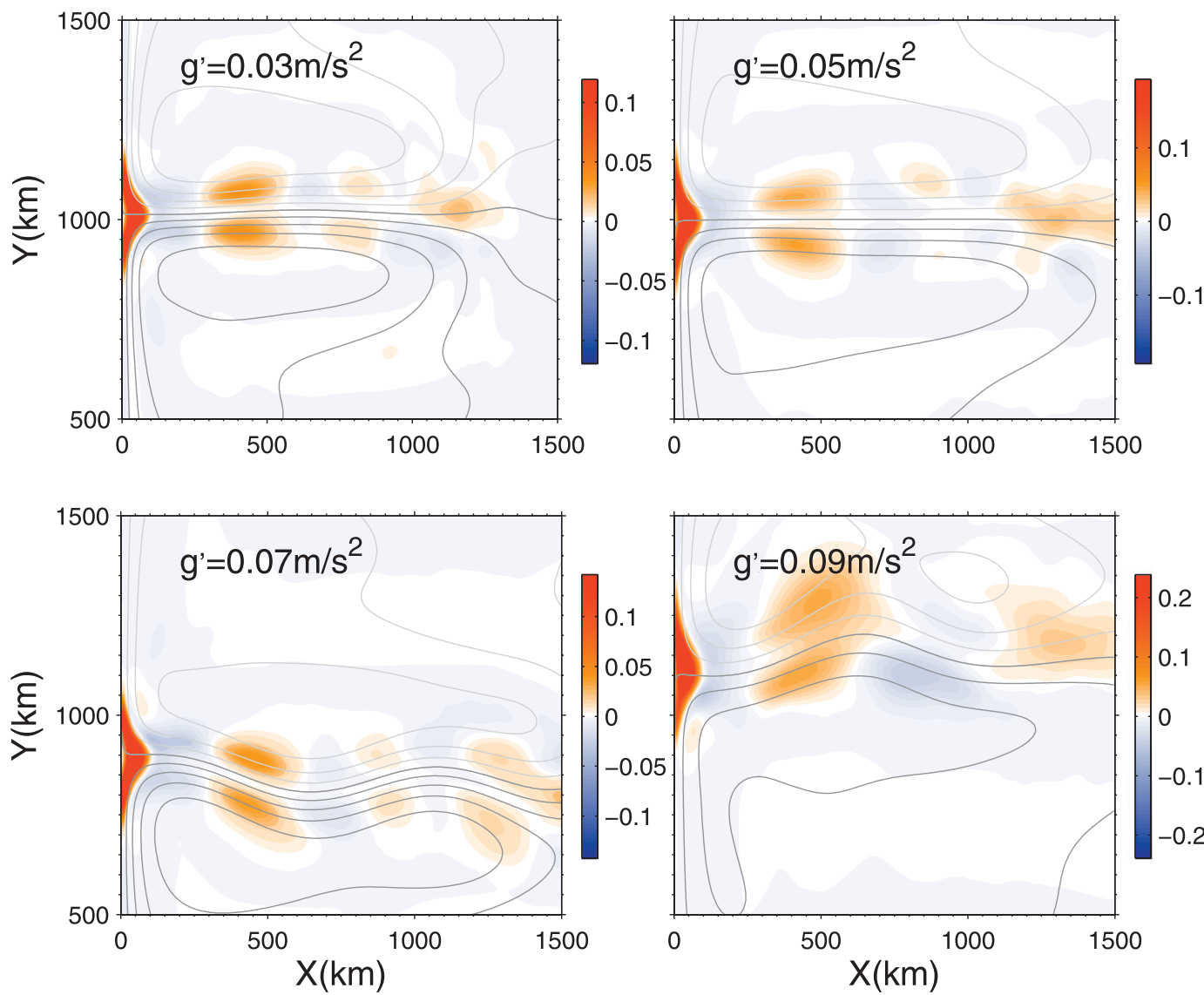

FIG. 8. The spatial distribution of the energy exchange between the mean flow and eddies as a result of work done by Reynolds force (positive values indicate that eddies extract energy from the mean flow; negative values indicate that the mean flow gets energy from eddies).

strong stratification regime (Fig. 10). This is consistent with the transition in the relative importance of T-RPV and T-TPV in the PV budget (Fig. 5). We note that $I_{P}$ increases with stratification, which indicates that the surface flow becomes progressively more barotropically unstable. In contrast to $I_{P}, I_{R}$ shows two distinct regimes: it changes little with $g^{\prime}$ in the weak stratification regime and decreases sharply in the strong stratification regime. This $g^{\prime}$ dependency is the same as for the variations of the bottom-layer circulation intensity (Fig. 2).

It is worth noting that the variation of the EKE in Fig. 6 is consistent with the variation of barotropical instability and baroclinic instability. Accordingly, the surface-layer EKE increases with the intensification of surface-layer barotropic instability when the stratification increases. The bottom-layer EKE, which is mainly associated with baroclinic instability, first increases $\left(g^{\prime} \leq\right.$ $\left.0.06 \mathrm{~m} \mathrm{~s}^{-2}\right)$ and then saturates $\left(g^{\prime} \geq 0.06 \mathrm{~m} \mathrm{~s}^{-2}\right)$ with increasing stratification.

In short, the saturation of the surface-layer circulation, to a large extent, can be attributed to the intensified barotropic instability. When the stratification becomes stronger, the surface-layer circulation intensifies (Fig. 2) because of the weakening of TPV in restraining the surface-layer inertial recirculation. As the surface-layer circulation is intensified, the shear of the EJ gets stronger and the surface flow becomes more subject to barotropic instability and releases energy to mesoscale eddies. With the increase in energetic eddies, the variability of the jet location amplifies, and so does the intergyre transport of PV.

\section{Bifurcation analysis}

In Fig. 11, we plot the standard deviations (STDs) of the EJ's location as a function of stratification calculated over the 20 years (year 80-100; section 4) versus over the total 200 years, respectively. It is shown that the $200-y r$ STD is much larger than the 20 -yr STD, especially in the strong stratification regime. And it is noted that the variation of the 200-yr STD is consistent with the variation of the 200-yr mean intergyre transport of RPV. 

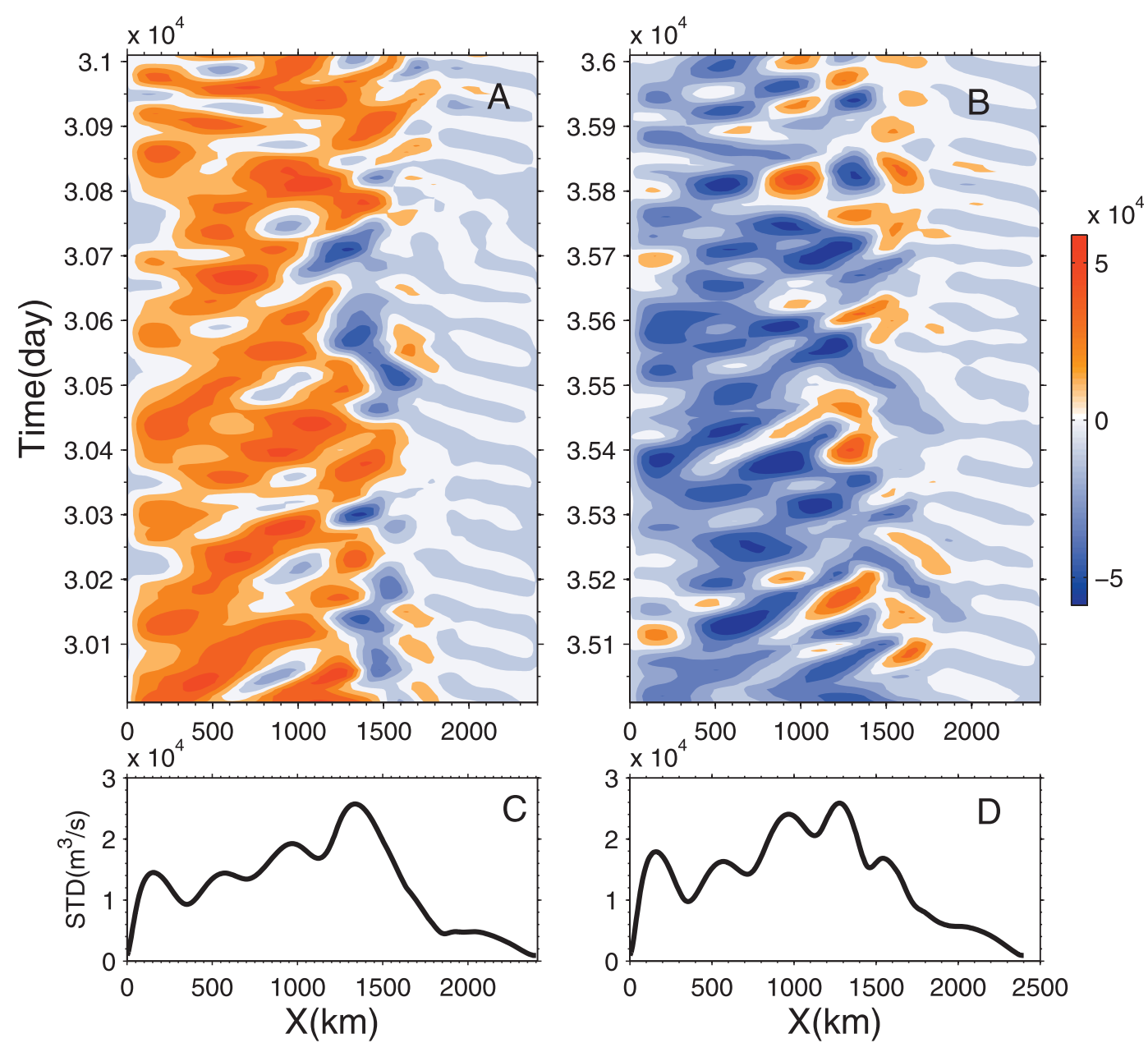

FIG. 9. The eastward-propagating signal. (top) Plots of $\varphi(x, t)$ averaged over $960<y<1040 \mathrm{~km}$ in meridional direction for (a) 30010-31000 days and (b) 35010-36000 days, the abscissa indicates the zonal location and the ordinate indicates the time [different patterns between (a) and (b) are due to the variability on decadal time scale as is illustrated in Fig. 13]. Eastward signals in both (a) and (b) are observed with a propagating time scale of 200 days at the jet region $(0<x<1500 \mathrm{~km})$. (bottom) Plots of STD of $\varphi(x, t)$ corresponding to (a),(b). It is observed that the variability of the jet flow peaks at the exit region of EJ at about $x=1300 \mathrm{~km}$.

In a three-layer QG model study, Nauw et al. (2004, hereafter N04) found that, as the lateral friction decreases, four regimes are encountered in the following order: the viscous antisymmetric regime, the asymmetric regime, the quasi-homoclinic regime, and the inertial antisymmetric regime. The emergence of asymmetric and quasi-homoclinic regimes will reduce the circulation intensity. Specifically, the former will reduce the PV input by the wind, and the latter will increase the intergyre transport of PV. Inspired by N04's work, we will in this section analyze the variability of the circulation to determine whether the regime shift occurs with increasing stratification that can contribute to the increased STD of the jet location.

With antisymmetric external forcing in the QG model, it is expected that the long-term-mean pattern should be also antisymmetric, and this is confirmed by the timemean streamfunction for $g^{\prime}$ less than $0.07 \mathrm{~m} \mathrm{~s}^{-2}$. However, as shown in Fig. 1, the time-mean streamfunction for $g^{\prime}$ larger than $0.07 \mathrm{~m} \mathrm{~s}^{-2}$ deviates from the antisymmetric pattern. In this case, either the subtropical gyre or the subpolar gyre shrinks in meridional size and increases in volume transport.

We define an asymmetry index for the mean flow as (Chang et al. 2001)

$$
\mathrm{AI}=\frac{\max \left(\left|\Psi_{\mathrm{tr}}\right|,\left|\Psi_{\mathrm{po}}\right|\right)}{\min \left(\left|\Psi_{\mathrm{tr}}\right|,\left|\Psi_{\mathrm{po}}\right|\right)}-1,
$$

where $\Psi_{\text {st }}$ and $\Psi_{\text {sp }}$ are defined as in section 2. If AI equals zero, the circulation is antisymmetric. Since asymmetric 

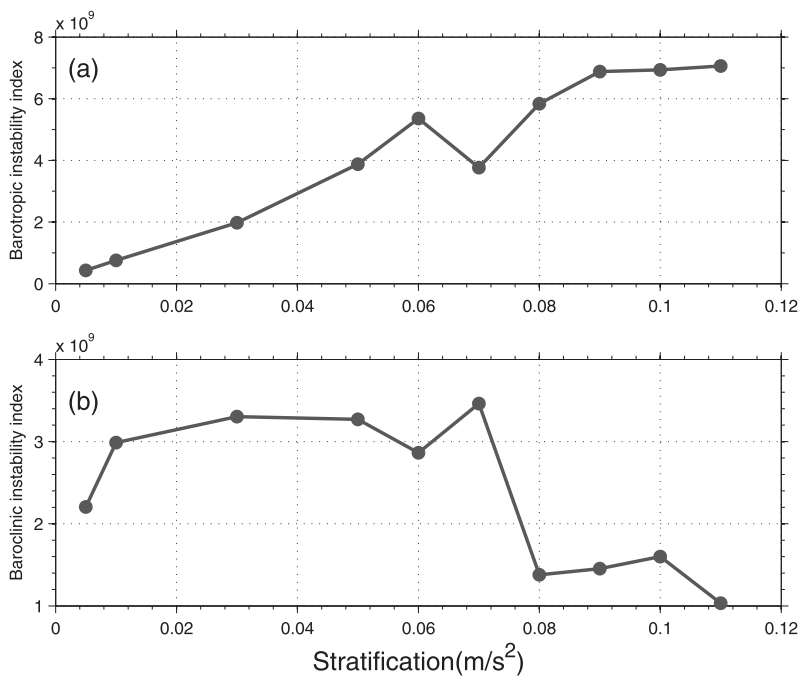

FIG. 10. Variation of the basin integrated energy that the perturbation extracts from the basic flow owing to (a) the surface-layer barotropic instability and (b) baroclinic instability (b).

solutions always coexist with a mirror symmetric solution (Jiang et al. 1995; Speich et al. 1995; Dijkstra and Ghil 1997), we do not distinguish between a southwardshifted jet versus a northward-shifted jet circulation in Eq. (14).

Within the weak stratification regime, the timemean circulations show exact antisymmetric patterns (Figs. 12a,b). However, with a further increase in stratification from $g^{\prime}=0.06 \mathrm{~m} \mathrm{~s}^{-2}$, AI increases until $g^{\prime}=$ $0.08 \mathrm{~m} \mathrm{~s}^{-2}$ where it reaches maximum and begins to decrease for larger $g^{\prime}$. Previous studies suggest that this emergence of asymmetry can be a result of the pitchfork bifurcation (Jiang et al. 1995, etc.). However, considering that both the EJ and circulation intensity are characterized by intense variability, we reckon that the asymmetry cannot be attributed to the pitchfork bifurcation, which is traditionally taken as a stationary bifurcation where branches of equilibrium points intersect (Seydel 2010). We calculate the percentage (\%) of time when the jet is shifted to the north of the midbasin axis and present the result in Fig. 12c. It can be seen that it is determined by how much time the jet spends on a jet-up state and jet-down state, where the jet-up (jet-down) state denotes that the jet is shifted to the north (south) of the midbasin axis.

In fact, the asymmetry discussed above is consistent with the introduction of a lower-frequency signal that may not be adequately sampled by the chosen timeaveraging interval. Figure 13 presents the variations of the 90-day-smoothed EJ's location for the total 200 years for the $g^{\prime}=0.03,0.05,0.07$, and $0.09 \mathrm{~m} \mathrm{~s}^{-2}$ cases. It is shown that, with increasing stratification, the variation of

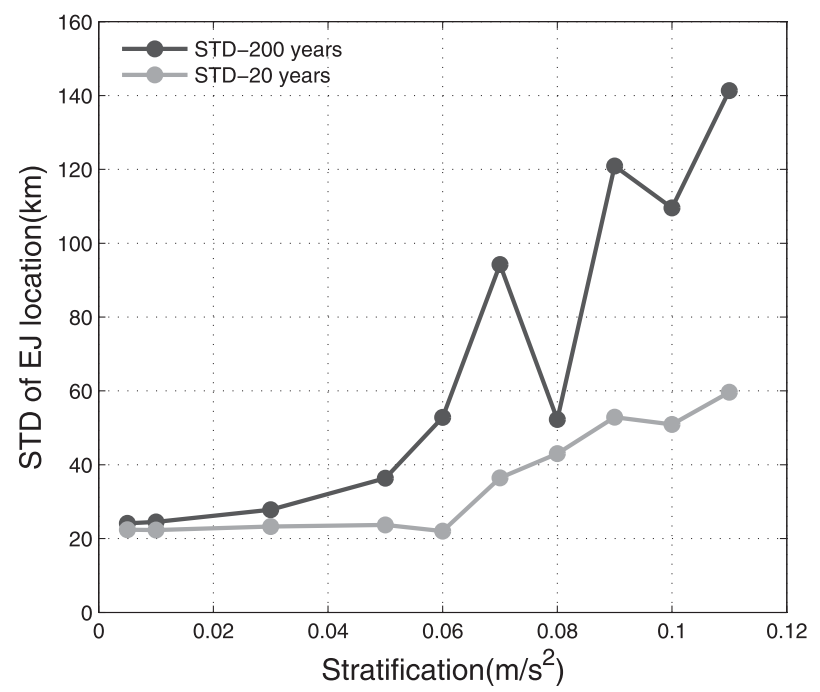

FIG. 11. Comparison of the STD of the EJ location for the 200 years, which includes switches between the jet-up state and jetdown state, and that for the chosen 20 years, which is carefully selected where no switch happens.

EJ's location undergoes three different patterns, two of which are quite similar to those observed in N04. Following N04, we name the first two regimes as inertial antisymmetric regime $\left(g^{\prime} \leq 0.03 \mathrm{~m} \mathrm{~s}^{-2}\right)$ and quasihomoclinic regime $\left(g^{\prime}=0.05 \mathrm{~m} \mathrm{~s}^{-2}\right)$. For $g^{\prime}$ larger than $0.06 \mathrm{~m} \mathrm{~s}^{-2}$, the variability of the jet location is characterized by switches between the jet-up and jet-down state on a time scale of multidecades. Here, we name this new pattern as the quasi-heteroclinic regime. Typical homoclinic and heteroclinic orbits can be found in Kuznetsov (1998, chapter 6). It is worth mentioning that the variation of the EJ location for $g^{\prime}<0.03 \mathrm{~m} \mathrm{~s}^{-2}$ resembles that for $g^{\prime}=0.03 \mathrm{~m} \mathrm{~s}^{-2}$, the variation of the EJ location for $g^{\prime}>0.05 \mathrm{~m} \mathrm{~s}^{-2}$ resembles that for $g^{\prime}=$ $0.07 \mathrm{~m} \mathrm{~s}^{-2}$, and $g^{\prime}=0.06 \mathrm{~m} \mathrm{~s}^{-2}$ represents a transition between the quasi-homoclinic and the quasi-heteroclinic regime (not shown).

Note that when the time-mean (shorter than the time scale over which the switches take place) jet is deviated from the midbasin axis, the input of PV by the wind is reduced and hence the circulation intensity will be restrained. Figure 11 compares the STD of the jet location for the chosen 20 years where no switches happen and that of the total 200 years, and it is shown that the switches contribute significantly to the variability of the jet location and consequently the intergyre transport of PV.

In summary, with the intensifying stratification, the circulation shifts from the inertial antisymmetric regime, to the quasi-homoclinic regime, and then to the quasiheteroclinic regime because of the successive system 

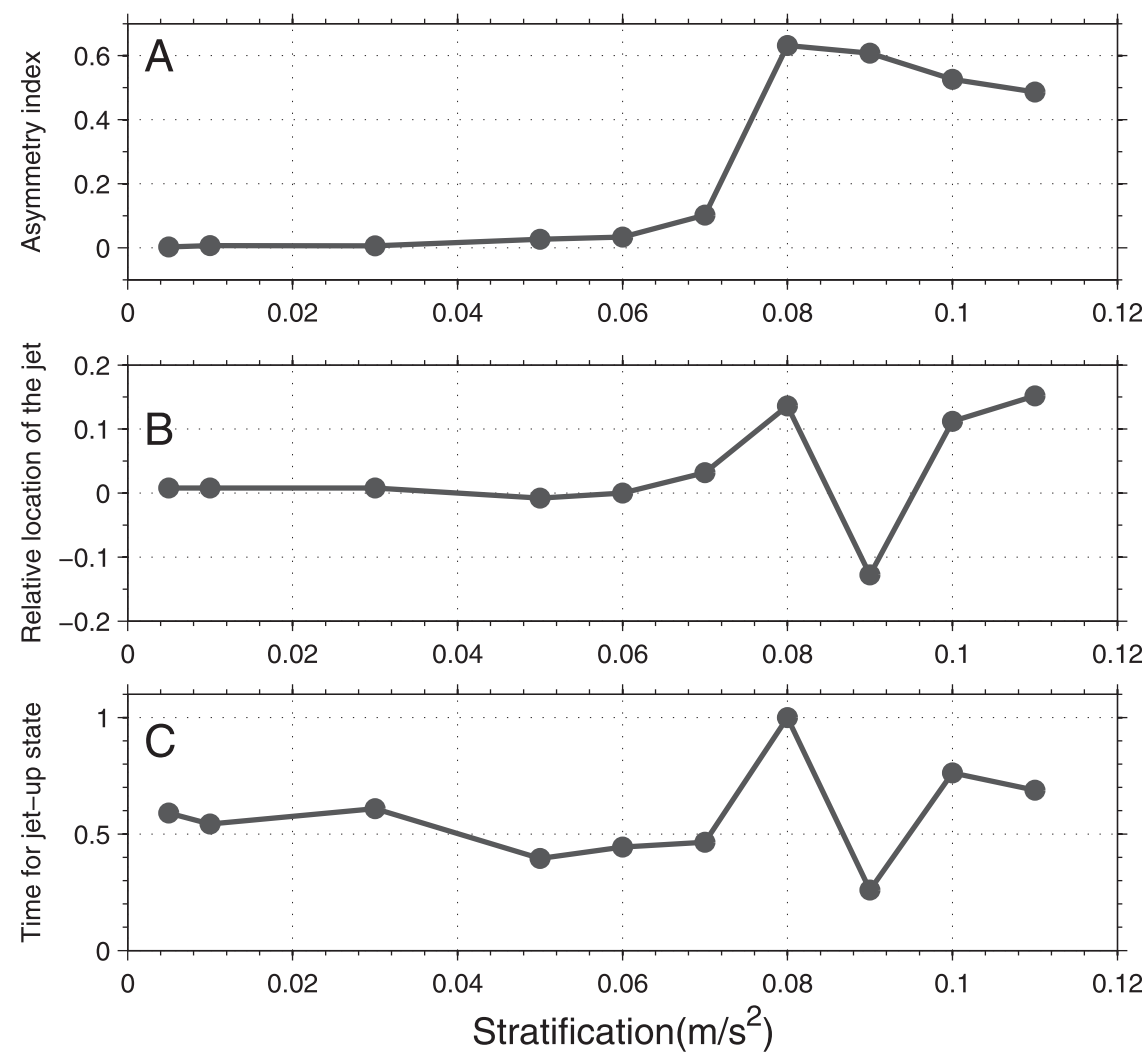

FIG. 12. (a) Variation of the asymmetric index (AI, nondimensional) for the long-term (200 yr) mean circulation with stratification; (b) the location (nondimensional) of the timemean EJ relative to $y=1000 \mathrm{~km}$ normalized by $L_{y} / 2$; and (c) variation of the time percent (\%) for the jet-up state with stratification (abscissa indicates $g^{\prime}$ in meters per square second).

bifurcations. The emergence of asymmetric states and the associated switches among different states contribute to the restraining of the surface-layer circulation intensity through both the reduction in the wind input $\mathrm{PV}$ and the increase in the intergyre transport of PV.

\section{Summary and further discussion}

In this study, we examine the response of the inertial recirculation to intensified stratification in a two-layer quasigeostrophic ocean circulation model. We extend the study by L97 and explore the strong nonlinear regime in which the weak nonlinear analysis becomes invalid.

By varying the reduced-gravity parameter characterizing the stratification, we find that the variation of the circulation intensity in the strong stratification regime differs from that in the weak stratification regime. Specifically, with increasing stratification, the surface-layer circulation is intensified in the weak stratification regime but saturates in the strong stratification regime. In comparison, the bottom-layer circulation is reduced weakly within the weak stratification regime and then sharply within the strong stratification regime. As a result, the barotropic transport by the double-gyre becomes increased for the weak stratification regime, conforming to the result in L97, but decreased for the further increase in stratification.

Through analyses of PV dynamics in the inertial recirculation region, it is found that TPV, which depends inversely on stratification, acts both to restrain the development of the surface-layer recirculation and to drive the bottom-layer flow. By increasing the stratification, the efficiency of TPV gets decreased and this strengthens the surface-layer circulation but weakens the bottom-layer circulation. The variation of the circulation in the weak stratification regime can be effectively explained by this PV dynamics.

To understand the behavior of the circulation in the strong stratification regime, we diagnose the PV budget of the circulation and conclude that it is the increased intergyre transport of RPV associated with the intensified variability of the EJ location that leads to the saturation of the surface-layer circulation, and hence the decrease of the barotropic transport.

Based on the instability analysis, it is found that eddies are generated at the confluence region of the 

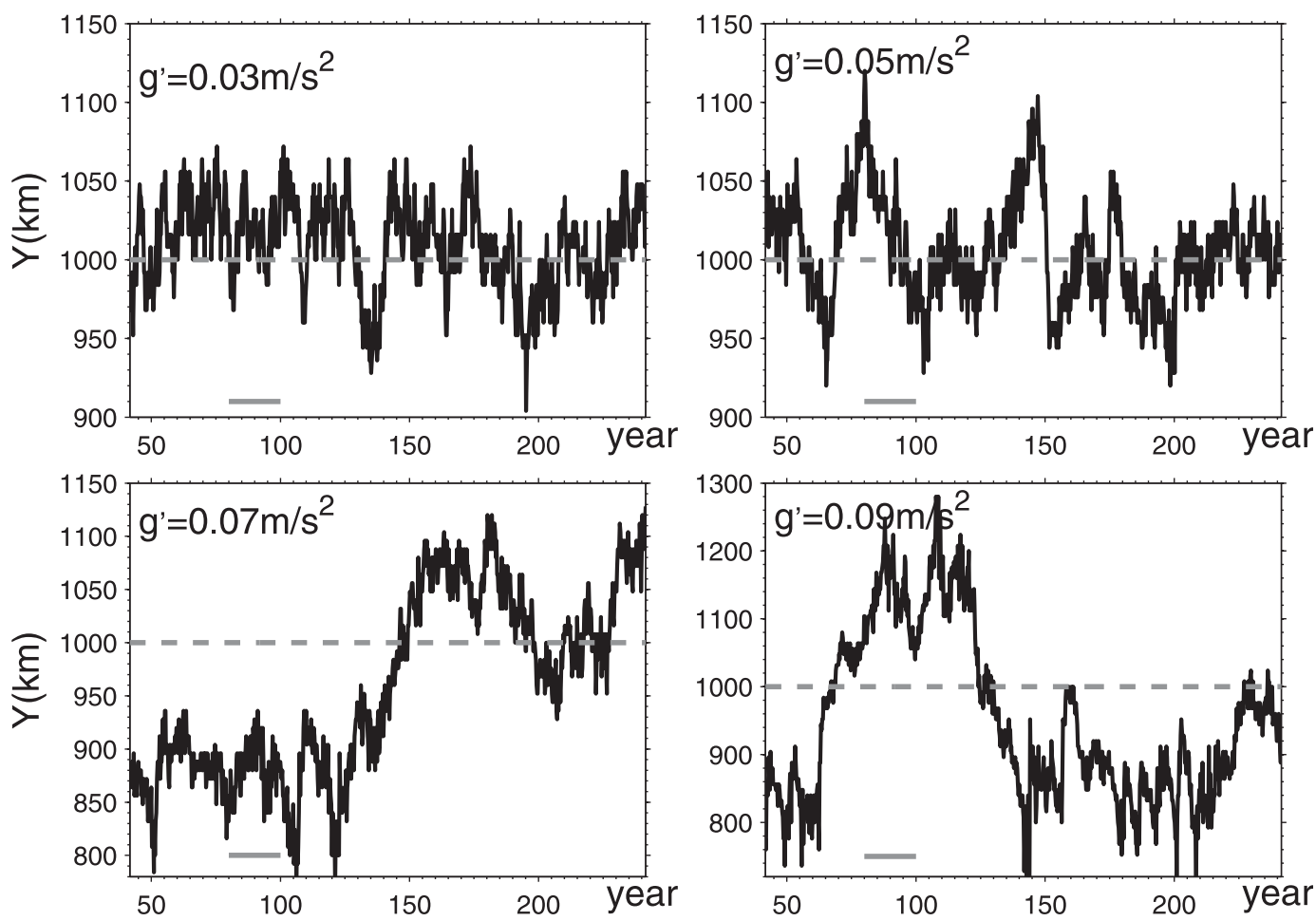

FIG. 13. Variation of the 90-day-smoothed jet location (solid black line) for the total 200 model years; The dashed gray line indicate the location of the midbasin axis; the solid gray line indicate the 20 years we choose where no switch between the jet-up and jet-down state occurs, specifically for $g^{\prime}>0.05 \mathrm{~m} \mathrm{~s}^{-2}$.

counterflowing WBCs as a result of barotropic instability. Advected eastward by the EJ, these eddies induce variability of the jet location and an intergyre transport of $\mathrm{PV}$. As the stratification is increased, the surface-layer circulation strengthens and the horizontal shear of surface flow becomes more intense. This brings about intensified barotropic instability and results in a variable circulation with excessive eddies and intense variability of the jet location. These processes lead to an enhanced intergyre transport of PV and a restrained circulation.

With the increased stratification, on time scales much longer than that associated with the barotropic and baroclinic instability, the circulation undergoes three different regimes: the inertial antisymmetric regime, the quasi-homoclinic regime, and the quasi-heteroclinic regime. These three regimes are characterized by differing variations of the jet location. The frequent switches among different states that characterize the quasihomoclinic and quasi-heteroclinic regimes contribute greatly to the STD of the jet location and therefore to the intergyre transport of PV. In addition, the emergence of asymmetry on a multidecadal time scale reduces the PV input by wind. Both of these effects contribute to damp the circulation intensity.

Temporally, the variability associated with the quasihomoclinic and quasi-heteroclinic regimes has decadal time scales, while the variability associated with the barotropic instability mechanism is likely to be shorter than a year. With respect to the emergence of quasihomoclinic and quasi-heteroclinic regimes, we attribute it to the system bifurcations and will leave the detailed examination into their mechanisms to future studies. In addition, we also recognize interannual signals in Fig. 13 that cannot be explained by either the barotropic instability or the bifurcation mechanisms. We hypothesize that these may be associated with Rossby basin modes that have the interannual time scales (Pedlosky 1987).

Given that the regimes shifts are associated with multidecadal time scales, the 200-yr study is far from being long enough to obtain a statistical view of this phenomenon. Sensitivity experiments (not shown) indicate that the time-mean circulation pattern depends on the time span to average, while the circulation intensity does not. Accordingly, if we make the time average to be sufficiently long, the preference for jet-up state or jetdown state disappears but the contribution to the variability of the jet location from regimes shift always remains. Therefore, another study with longer time integration on the statistics of regime shifts is expected to perfect this research.

Finally, some comments are required on what will happen if the stratification is further increased. It cannot 
be guaranteed that the barotropic instability and the regime shift mechanism will persist, so that the increased intergyre transport of potential vorticity can maintain the saturation of the surface-layer circulation. In fact, our sensitivity experiments in the basin of a smaller zonal scale reveal that another regime characterized by the inertial runaway phenomenon comes into existence when the stratification exceeds a critical value. This is a topic we plan to explore further in our future studies.

Acknowledgments. This work is supported by National Major Research Plan of Global Change (2013CB956201), and Chinese National Science Foundation Key Research Project (41130859). The authors wish to thank Dr. Linhao Zhong for providing the quasigeostrophic model used in this study. Comments from the anonymous reviewers are greatly appreciated.

\section{REFERENCES}

Beliakova, N., 1998: Generation and maintenance of recirculation by Gulf Stream instabilities. Ph.D. thesis, Massachusetts Institute of Technology/Woods Hole Oceanographic Institute Joint Program, 224 pp.

Berloff, P., 2005: On the rectification of randomly forced flows. J. Mar. Res., 63, 497-527.

— circulation. J. Mar. Res., 56, 937-993.

—, A. McC. Hogg, and W. Dewar, 2007: The turbulent oscillator: A mechanism of low-frequency variability of the wind-driven ocean gyres. J. Phys. Oceanogr., 37, 2363-2386.

Capotondi, A., M. A. Alexander, N. A. Bond, E. N. Curchitser, J. D. Scott, 2012: Enhanced upper ocean stratification with climate change in the CMIP3 models. J. Geophys. Res., 117, C04031, doi:10.1029/2011JC007409.

Cessi, P., 1988: A stratified model of the inertial recirculation. J. Phys. Oceanogr., 18, 662-682.

—, and G. R. Ierley, 1995: Symmetry-breaking multiple equilibira in quasigeostrophic, wind-driven flows. J. Phys. Oceanogr., 23, 1196-1205.

Chang, K.-I., M. Ghil, K. Ide, and C. C. Aaron Lai, 2001: Transition to aperiodic variability in a wind-driven double-gyre circulation model. J. Phys. Oceanogr., 31, 1260-1286.

Cravatte, S., T. Delcroix, D. Zhang, M. J. McPhaden, and J. Leloup, 2009: Observed freshening and warming of the western Pacific Warm Pool. Climate Dyn., 33, 565-589.

Deser, C., A. S. Phillips, and M. A. Alexander, 2010: Twentieth century tropical sea surface temperature trends revisited. Geophys. Res. Lett., 37, L10701, doi:10.1029/2010GL043321.

Dijkstra, H. A., 2000: Nonlinear Physical Oceanography. Kluwer Academic Publishers, 480 pp.

_ , and M. Ghil, 2005: Low-frequency variability of the largescale ocean circulation: A dynamical approach. Rev. Geophys., 43, RG3002, doi:10.1029/2002RG000122.

Fofonoff, N. P., 1954: Steady flow in a frictionless homogeneous ocean. J. Mar. Res., 13, 254-262.
Haidvogel, D., J. McWilliams, and P. Gent, 1992: Boundary current separation in a qusi-geostrophic, eddy-resolving ocean circulation model. J. Phys. Oceanogr., 22, 882-902.

Hogg, N. G., 1983: A note on the deep circulation of the west North Atlantic Ocean. Deep-Sea Res., 30, 945-961.

Ierley, G. R., 1987: On the onset of inertial recirculation in barotropic general circulation models. J. Phys. Oceanogr., 17, 2366-2374.

Jayne, S. R., and N. Hogg, 1999: On recirculation forced by an unstable jet. J. Phys. Oceanogr., 29, 2711-2718.

— , N. G. Hogg, and P. Malanotte-Rizzoli, 1996: Recirculation gyres forced by a beta-plane jet. J. Phys. Oceanogr., 26, 492-504.

Jiang, S., F.-F. Jin, and M. Ghil, 1995: Multiple equilibria and aperiodic solutions in a wind-driven double gyre, shallow water model. J. Phys. Oceanogr., 25, 764-786.

Kuznetsov, Y. A., 1998: Elements of Applied Bifurcation Theory. 2nd ed. Springer-Verlag, $614 \mathrm{pp}$.

Liu, Z., 1997: The influence of stratification on the inertial recirculation. J. Phys. Oceanogr., 27, 926-940.

Marshall, D., and J. Marshall, 1992: Zonal penetration scale of midlatitude oceanic jets. J. Phys. Oceanogr., 22, 1018-1032.

Marshall, J., and A. Nurser, 1986: Steady, free circulation in a stratified quasigeostrophic ocean. J. Phys. Oceanogr., 16, 1799-1813.

McCalpin, J. D., and D. B. Haidvogel, 1996: Phenomenology of the low-frequency variability in a reduced gravity quaigeostrophic double-gyre model. J. Phys. Oceanogr., 26, 739-752.

Nauw, J. J., H. A. Dijkstra, and E. Simonnet, 2004: Regimes of low-requency variability in a three layer quasigeostrophic ocean model. J. Mar. Res., 62, 685-720.

Pedlosky, J., 1987: Geophysical Fluid Dynamics. 2nd ed. SpringerVerlag, 710 pp. , 1996: Ocean Circulation Theory. Springer-Verlag, 452 pp.

Pierini, S., H. D. Dijkstra, and A. Riccio, 2009: A nonlinear theory of the Kuroshio extension bimodality. J. Phys. Oceanogr., 39, 2212-2229.

Primeau, F. W., 1998: Multiple equilibria and low-frequency variability of wind-driven ocean models. Ph.D. thesis, Massachusetts Institute of Technology/Woods Hole Oceanographic Institute Joint Program, 158 pp.

Qiu, B., and W. Miao, 2000: Kuroshio path variations south of Japan: Bimodality as a self-sustained internal oscillation. J. Phys. Oceanogr., 30, 2124-2137.

, S. Chen, P. Hacker, N. Hogg, S. Jayne, and H. Sasaki, 2008: The Kuroshio extension northern recirculation gyre: Profiling float measurements and forcing mechanism. J. Phys. Oceanogr., 38, 1764-1779.

Schmitz, W. J., 1980: Weakly-depth-dependent segments of the Atlantic circulation. J. Mar. Res., 38, 111-133.

Seydel, R., 2010: Practical Bifurcation and Stability Analysis. 3rd ed. Springer-Verlag, 493 pp.

Sheremet, V. A., G. R. Ierley, and V. M. Kamenkovich, 1997: Eigenanalysis of the two-dimensional wind-driven ocean circulation problem. J. Mar. Res., 55, 57-92.

Speich, S., H. A. Dijksra, and M. Ghil, 1995: Successive bifurcations in a shallow water model applied to the wind-driven ocean circulation. Nonlinear Processes Geophys., 2, 241-268.

Waterman, S., and S. R. Jayne, 2009: Eddy-mean flow interaction in the along-stream development of a western boundary current jet: An idealized model study. J. Phys. Oceanogr., 41, 681-707. 